\title{
Article
}

\section{Peanut-shaped metallicity distributions in bulges of edge-on galaxies: the case of NGC 4710}

Gonzalez, Oscar A., Debattista, Victor P, Ness, Melissa, Erwin, Peter and Gadotti, Dimitri A.

Available at http://clok.uclan.ac.uk/16806/

Gonzalez, Oscar A., Debattista, Victor P ORCID: 0000-0001-7902-0116, Ness, Melissa, Erwin, Peter and Gadotti, Dimitri A. (2016) Peanut-shaped metallicity distributions in bulges of edge-on galaxies: the case of NGC 4710. Monthly Notices of the Royal Astronomical Society: Letters, 466 (1). L93-L97. ISSN $1745-3933$

It is advisable to refer to the publisher's version if you intend to cite from the work. http://dx.doi.org/10.1093/mnrasl/slw232

For more information about UCLan's research in this area go to http://www.uclan.ac.uk/researchgroups/ and search for <name of research Group>.

For information about Research generally at UCLan please go to http://www.uclan.ac.uk/research/

All outputs in CLoK are protected by Intellectual Property Rights law, including Copyright law. Copyright, IPR and Moral Rights for the works on this site are retained by the individual authors and/or other copyright owners. Terms and conditions for use of this material are defined in the policies page. 


\title{
Peanut-shaped metallicity distributions in bulges of edge-on galaxies: the case of NGC 4710
}

\author{
Oscar A. Gonzalez, ${ }^{1 \star}$ Victor P. Debattista, ${ }^{2}$ Melissa Ness, ${ }^{3}$ Peter Erwin ${ }^{4}$ \\ and Dimitri A. Gadotti ${ }^{5}$ \\ ${ }^{1}$ UK Astronomy Technology Centre, Royal Observatory, Blackford Hill, Edinburgh EH9 3HJ, UK \\ ${ }^{2}$ Jeremiah Horrocks Institute, University of Central Lancashire, Preston PR1 2HE, UK \\ ${ }^{3}$ Max-Planck-Institut fur Astronomie, Konigstuhl 17, D-69117 Heidelberg, Germany \\ ${ }^{4}$ Max-Planck-Insitut für extraterrestrische Physik, Giessenbachstrasse, D-85748 Garching, Germany \\ ${ }^{5}$ European Southern Observatory, Ave. Alonso de Cordova 3107, Casilla 19, 19001, Santiago, Chile
}

Accepted 2016 November 9. Received 2016 November 9; in original form 2016 October 7

\begin{abstract}
Bulges of edge-on galaxies are often boxy/peanut-shaped (B/PS), and unsharp masks reveal the presence of an X shape. Simulations show that these shapes can be produced by dynamical processes driven by a bar which vertically thickens the centre. In the Milky Way, which contains such a B/PS bulge, the X-shaped structure is traced by the metal-rich stars but not by the metal-poor ones. Recently, Debattista et al. interpreted this property as a result of the varying effect of the bar on stellar populations with different starting kinematics. This kinematic fractionation model predicts that cooler populations at the time of bar formation go on to trace the $\mathrm{X}$ shape, whereas hotter populations are more uniformly distributed. As this prediction is not specific to the Milky Way, we test it with Multi Unit Spectroscopic Explorer (MUSE) observations of the B/PS bulge in the nearby galaxy NGC 4710. We show that the metallicity map is more peanut-shaped than the density distribution itself, in good agreement with the prediction. This result indicates that the $\mathrm{X}$-shaped structure in B/PS bulges is formed of relatively metal-rich stars that have been vertically redistributed by the bar, whereas the metal-poor stars have a more uniform, box-shaped distribution.
\end{abstract}

Key words: galaxies: bulges-galaxies: evolution-galaxies: formation-galaxies: stellar content-galaxies: structure.

\section{INTRODUCTION}

The study of the bulges of spiral galaxies is of fundamental importance for obtaining a complete understanding of the processes involved in the formation and evolution of disc galaxies. Early galaxy mergers are thought to be very efficient in producing spheroids in the central regions of disc galaxies (Brooks \& Christensen 2016). These components, known as classical bulges, would have similar properties to scaled-down elliptical galaxies (Kormendy \& Kennicutt 2004; Hopkins et al. 2010). However, observationally, the central regions of galaxies are commonly found to be dominated by stellar bars (e.g. Lütticke, Dettmar \& Pohlen 2000). Furthermore, disc galaxies seen edge-on often show central bulges that appear boxy, or sometimes peanut- or X-shaped. Numerical simulations provide clues to the nature of these systems placing them in the family of secular products arising from the vertical heating and thickening of bars. Observations have confirmed the link of this

\footnotetext{
^E-mail: oscar.gonzalez@stfc.ac.uk
}

kind of bulges to the presence of stellar bars (see Athanassoula 2016, for a detailed review). These boxy/peanut-shaped (B/PS) bulges are produced either by the buckling instability of bars (Raha et al. 1991) or by resonant trapping (Quillen et al. 2014). B/PS bulges are very common (Laurikainen \& Salo 2016) and even our own Milky Way (MW) Galaxy has a B/PS bulge (Zoccali \& Valenti 2016, and references therein).

Secular evolution via bar buckling readily produces bulges with the kinematics (e.g. Shen et al. 2010) and X-shaped morphology (e.g. Wegg \& Gerhard 2013) of the MW's bulge. However, more detailed studies of the stellar populations of the MW's B/PS bulge have produced unexpected results in terms of a vertical metallicity gradient (Zoccali et al. 2008; Gonzalez et al. 2013; Johnson et al. 2013), an X-shape that is traced by the more metal-rich stars, but not by the metal-poor ones (e.g. Ness et al. 2013; Vásquez et al. 2013), and a nearly axisymmetric distribution of RR-Lyrae variables at $b \simeq 4^{\circ}$ (Dékány et al. 2013). These properties of the MW's bulge seemed to require the additional presence of a classical bulge. Recently, Debattista et al. (2016) resolved this discrepancy in the secular evolution scenario by demonstrating how bars are able 
to produce a separation between different stellar populations during the $\mathrm{B} / \mathrm{PS}$ bulge formation. Because this separation is induced by the different initial in-plane kinematics (which are correlated with ages and therefore with metallicity), they adopted the term kinematic fractionation to refer to it. They showed that the properties of the resulting B/PS bulges nicely follow the trends observed in the MW.

Kinematic fractionation is not specific to the MW. One testable prediction of kinematic fractionation is that $\mathrm{B} / \mathrm{PS}$ bulges observed in edge-on disc galaxies will show metallicity maps that trace a peanut shape, analogous to the X-shape seen in the MW. In this context, NGC 4710 is one of the best laboratories to test this prediction. NGC 4710 , located at $16.9 \mathrm{Mpc}$, has a stellar mass of $M_{\star} \sim 7 \times 10^{10} \mathrm{M}_{\odot}$ (Cappellari et al. 2013), is classified as an exactly edge-on barred galaxy with a B/PS bulge (Buta et al. 2015), and has no co-spatial large-scale classical bulge (Gadotti 2012; Gonzalez et al. 2016). Furthermore, the arms of its X-shaped bulge structure are seen very prominently, as a consequence of a nearly side-on orientation of the bar (Buta et al. 2015). In this Letter, we investigate the stellar population properties of the B/PS bulge of NGC 4710, directly testing the prediction of kinematic fractionation.

\section{OBSERVATIONS}

We obtained spectral and imaging coverage of NGC 4710 using the Multi Unit Spectroscopic Explorer (MUSE) instrument installed on the Very Large Telescope (VLT). The observations were taken as part of the MUSE Science Verification observing run in 2014 June and their details can be found in the study of the kinematics of NGC 4710 presented in Gonzalez et al. (2016). MUSE (Bacon et al. 2010) is an optical wide-field integral-field spectrograph that uses the image slicing technique to cover a field of view of $1 \operatorname{arcmin} \times 1$ arcmin in wide-field mode resulting in a spatial sampling of $0.2 \operatorname{arcsec} \times 0.2$ arcsec spaxels and resolving power from $R \sim 2000$ at $4600 \AA$ to $R \sim 4000$ at $9300 \AA$. The full field is split up into 24 subfields that are fed into one of the 24 integral field units (IFUs) of the instrument. Observations of NGC 4710 were carried without adaptive optics at the nominal wavelength range (4650-9300 $\AA$ ). The central coordinates of the observed field $\left(\alpha=12^{\mathrm{h}} 49^{\mathrm{m}} 37^{\mathrm{s}} .9, \delta=+15^{\circ} 10^{\prime} 00^{\prime} .8, \mathrm{~J} 2000\right)$ were optimized as described in Gonzalez et al. (2016) to cover the inner part of the B/PS bulge and to extend the coverage to the expected limit of the bulge on the north-west side of the galaxy.

\section{SIMULATION}

In this study, we investigate the stellar population properties of the B/PS bulge of NGC 4710 by comparing them to a star-forming $\mathrm{N}$-body chemodynamical simulation which evolved to form a B/PS bulge. The simulation was presented by Cole et al. (2014) and Ness et al. (2014), while a full description of the physical processes involved was given in Debattista et al. (2016). In the simulation, a disc galaxy forms purely out of gas cooling from a spherical corona that settles into a disc, triggering continuous star formation. The galaxy forms a strong bar after $2 \mathrm{Gyr}$ and by $10 \mathrm{Gyr}$, it has formed a B/PS bulge (Debattista et al. 2016). Very important for our study is the fact that all stars in the simulation form from gas, rather than being put by hand as typically done in $\mathrm{N}$-body simulations. Thus, the simulation was able to follow the chemical evolution of all stellar populations.

\section{STELLAR POPULATIONS ANALYSIS}

Gonzalez et al. (2016) used the MUSE observations of NGC 4710 to map the kinematics of its bulge. They used the Penalized Pixel Fitting routine $\operatorname{PPXF}^{1}$ (Cappellari \& Emsellem 2004) to evaluate the galaxy's stellar kinematics by fitting the single stellar population (SSP) MILES library of Vazdekis et al. (2010) to the observed spectra in pixel space using a maximum penalized likelihood method. The line-of-sight (LOS) mean velocity and velocity dispersion were obtained by fitting templates to the observed spectra in each spatial bin.

In this work, we extend the analysis of Gonzalez et al. (2016) to investigate the stellar populations of the bulge of NGC 4710 . We perform full-spectrum fitting with PPXF using the; MIUSCAT library $^{2}$ of SSP models (Vazdekis et al. 2012). The SSP models from the MIUSCAT library have been computed theoretically from the MILES and CaT empirical spectra (Cenarro et al. 2001) and were preferred for our study as they have been shown to accurately reproduce the continuum and line profiles, which are critical for the analysis of stellar populations. We select a grid with ages ranging from 1 to $14.1 \mathrm{Gyr}$ and metallicities ${ }^{3}$ ranging from -1.31 to +0.22 dex, computed using a Salpeter (1955) initial mass function (unimodal with slope 1.3) and based on the Padova isochrones from Girardi et al. (2000).

We follow the same methodology as in Gonzalez et al. (2016) and use PPXF to find the best-fitting template spectrum over each spatial bin over the wavelength range (4750-5550 ̊̊) to avoid regions potentially contaminated by residuals of strong sky emission lines. This spectral region has been used in similar analysis by the SAURON (Bacon et al. 2001) and ATLAS ${ }^{3 \mathrm{D}}$ (Cappellari et al. 2011) surveys, as it includes strong stellar population indicators such as $\mathrm{H} \beta$ (sensitive to age) as well as Mgb and Fe5250 (sensitive to metallicity).

Gas emission lines are not masked, unlike in the earlier kinematic analysis (Gonzalez et al. 2016), in order to include the absorption $\mathrm{H} \beta$ line in the analysis. Instead, we perform a two component fit in PPXF including the MIUSCAT templates as well as a set of Gaussian emission templates. Further, we allow PPXF to treat the fit of the emission-line template as a different kinematic component to account for possible decoupled kinematics between gas and stellar components. In order to accurately perform the two-component fit, we increase the binning with respect to the kinematic study (minimum $\mathrm{S} / \mathrm{N}=50$ ) by imposing a minimum $\mathrm{S} / \mathrm{N}$ of 100 in each spatial bin using the Voronoi-binning method (Cappellari \& Copin 2003).

We use only multiplicative polynomials to account for flux calibration errors without including any additive polynomials. As described in Cappellari \& Emsellem (2004), using additive polynomials is recommended when fitting the LOS velocity distribution but they should be avoided when inferring stellar populations as they can affect the spectral line strength and bias the metallicity and age calculations.

We set PPXF to find an optimal spectral fitting solution based on the weights applied to each template spectrum from the MIUSCAT library in the age-metallicity grid using a regularization parameter. As described in Guérou et al. (2016), the statistical consistency of the best-fitting solution to the data can be optimized by increasing

\footnotetext{
${ }^{1}$ http://www-astro.physics.ox.ac.uk/ mxc/software/

${ }^{2}$ http://miles.iac.es/pages/stellar-libraries/miles-library.php

${ }^{3}$ We use the MIUSCAT base models, which are computed using stars selected on the basis of their $[\mathrm{Fe} / \mathrm{H}]$ metallicity. Therefore, these models follow the abundance pattern of the MW.
} 

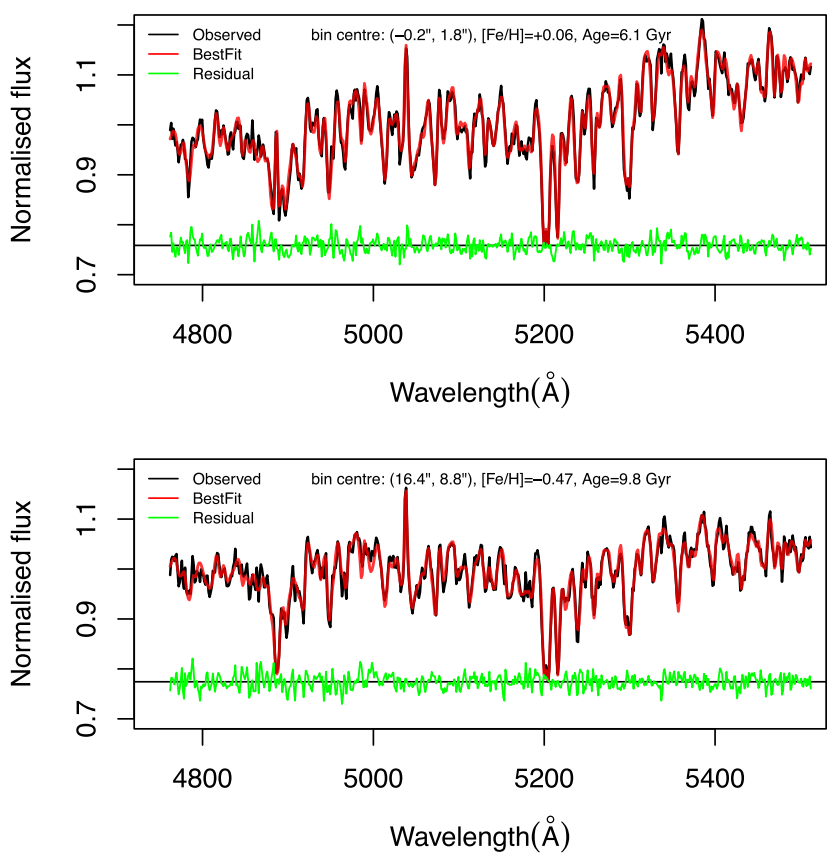

Figure 1. Stellar population solutions of PPXF from the full spectral-fitting procedure for two representative regions of NGC 4710 . The black lines show the observed, integrated spectra for two spatial bins with $\mathrm{S} / \mathrm{N} \sim 100$ centred in the disc plane (upper panel) and in the outer bulge (lower panel) along the minor axis. The best-fitting template spectra obtained from PPXF using the MIUSCAT libraries and emission-line templates are shown as red lines. The residuals from the fits for each case are shown in green.

the regularization parameter until the $\chi^{2}$ value of the template fit exceeds that of the unregularized fit by $\sqrt{2 N}$, where $N$ is the number of spaxels. In this way, we ensure that the regularization procedure constrains the solution such that the weights assigned to neighbouring age and metallicity templates changes smoothly while being fully consistent with our observed spectrum.

The final representative stellar population of the observed integrated spectrum for each bin is then obtained by calculating a weighted average of the metallicities and ages of the grid using the weights calculated by PPXF. Fig. 1 shows the best-fitting so- lution for two spatial bins of the MUSE cube. The MIUSCAT template models are normalized to a star of one solar mass so the resulting mean age and metallicity values are mass-weighted. We estimate the uncertainties in our measurements by assuming symmetry with respect to the minor axis of the galaxy and evaluating the standard deviation of the $[\mathrm{Fe} / \mathrm{H}]$ difference at symmetric locations. We find a typical error of $\sim 0.03 \mathrm{dex}$ in the mean $[\mathrm{Fe} / \mathrm{H}]$ and 0.8 Gyr for the mean stellar age. These values are expected to be slightly larger in the outermost bins that are located furthest from the mid-plane.

\subsection{Age and metallicity maps of NGC 4710}

Fig. 2 shows the resulting mass-weighted metallicity and age maps of the bulge of NGC 4710. The maps suggest the presence of a thin disc component of near-solar metallicity with a mean age of 5-6 Gyr and they show that there is a clear vertical metallicity gradient. We note that there is a region near the mid-plane (but clearly not in the centre of the galaxy) dominated by younger stellar populations $(2 \mathrm{Gyr})$ than the rest of the disc, which coincides with a flux excess seen in the reconstructed image. In Gonzalez et al. (2016), this region was identified as a stellar population located in the foreground disc because of its distinct kinematics and strong gas emission lines. The fact that the stellar population in this region is also very young and metal-poor with respect to the rest of the galaxy supports this conclusion and, furthermore, suggests that this is a recently triggered star-forming region.

At larger heights from the plane, in the region dominated by the $\mathrm{B} / \mathrm{PS}$ bulge, the mean age of the stellar population quickly becomes older, of the order of 8-9 Gyr, while the mean metallicity decreases to $[\mathrm{Fe} / \mathrm{H}]=-0.2$. Beyond this point, the age map is consistent with a predominantly old population with a mean age of $10 \mathrm{Gyr}$, while the vertical metallicity gradient extends to $\sim 35 \operatorname{arcsec}(\sim 2.0 \mathrm{kpc}$ at $16.9 \mathrm{Mpc})$ from the plane of the galaxy, reaching a metallicity of $[\mathrm{Fe} / \mathrm{H}]=-0.8$ dex in the region of the outer bulge and halo.

The most striking feature of the mass-weighted metallicity map of NGC 4710 is its peanut shape, that is, the vertical metallicity gradient has a different pattern along the minor axis than at the sides of the B/PS bulge. The metal-rich population extends to larger heights away from the plane at intermediate distances $(\sim 1.5 \mathrm{kpc})$ from the centre than it does along the minor axis. We
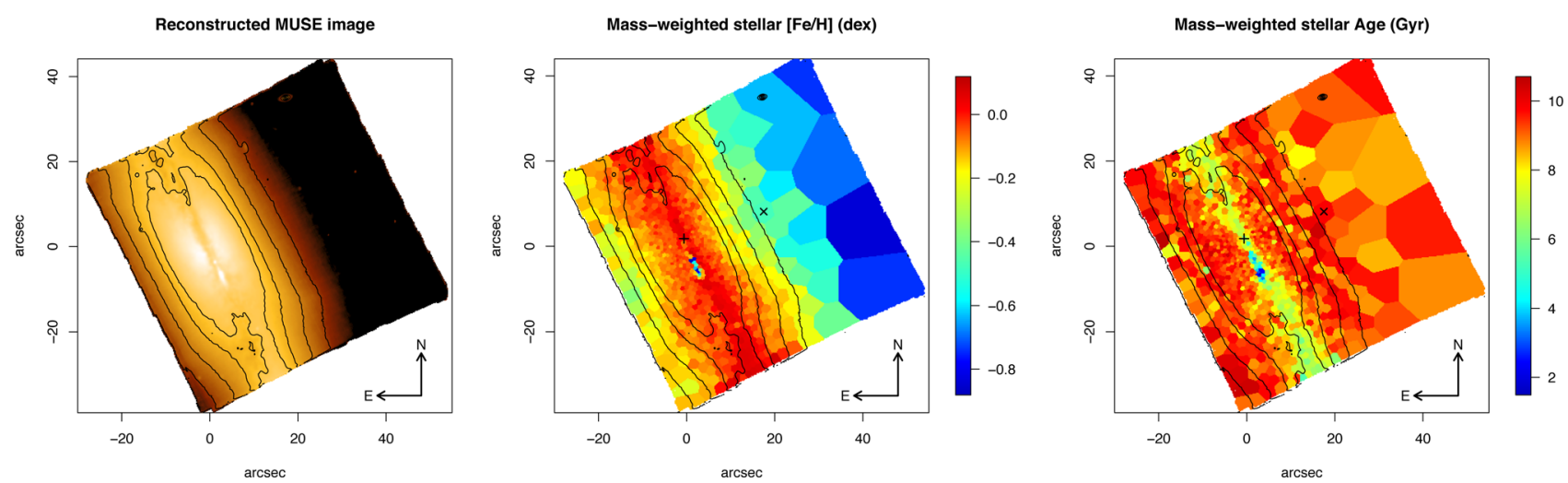

Figure 2. The left-hand panel shows the reconstructed flux map and isophote contours of the MUSE observations of NGC 4710 in arbitrary flux units. The mass-weighted stellar population maps of NGC 4710 resulting from the regularized spectral fitting using PPXF are shown in the middle and right-hand panels for metallicity and age, respectively. Isophote contours are overplotted for reference to the regions mapped in the reconstructed image. The locations of the spectra displayed in the upper and lower panels of Fig. 1 are shown as + and $\times$, respectively. 

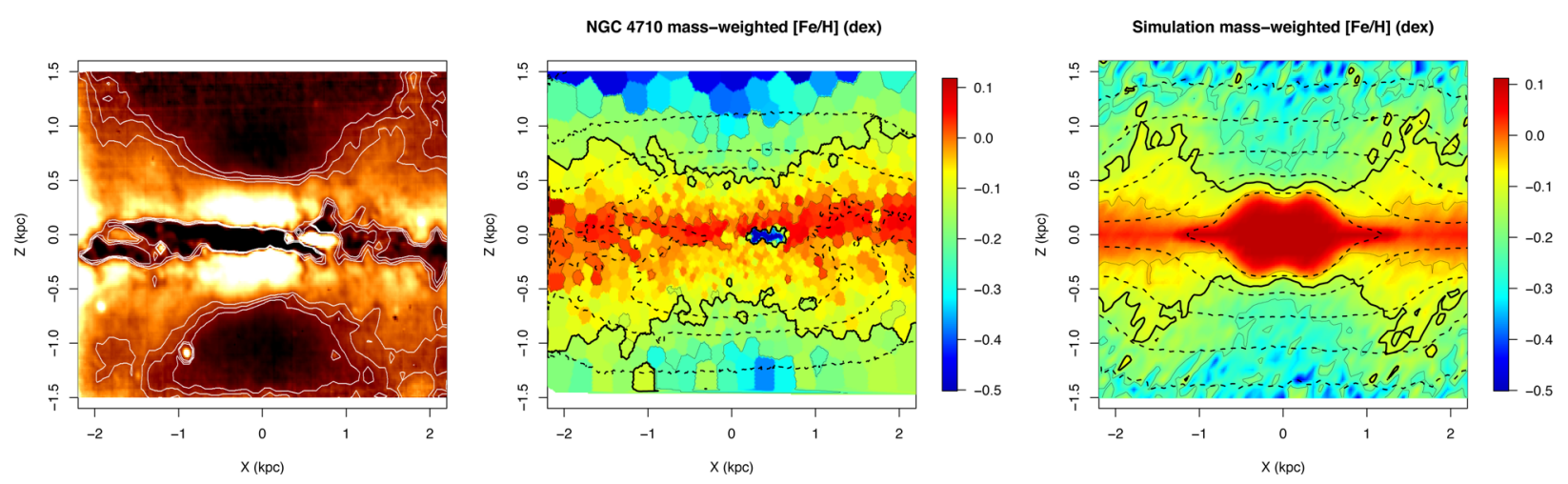

Figure 3. The left-hand panel shows the unsharp-masked MUSE image of NGC 4710 obtained by dividing the original image by a smoothed one using a Gaussian filter with $\sigma=15$ pixels. The middle panel shows the mass-weighted metallicity map of NGC 4710. Dashed contour lines are stellar surface brightness isophotes, as in Fig. 2. The right-hand panel shows the metallicity map of the simulation from Debattista et al. (2016). The density contours of the simulation are also shown in the right-hand panel as dashed lines. The spatial coordinates of the simulation were scaled by a factor of $1.2 \mathrm{and}$ the [Fe/H] values in the simulation were decreased by $0.35 \mathrm{dex}$ for comparison. The colour scale saturates at $[\mathrm{Fe} / \mathrm{H}]=0.1 \mathrm{dex}$ for visualization. We assume a distance to NGC 4710 of $16.9 \mathrm{Mpc}$. The peanut shape of the metallicity maps is outlined by the boldfaced contour at $[\mathrm{Fe} / \mathrm{H}]=-0.1$ for both NGC 4710 and the simulation.

refer to this gradient pattern as the peanut-shaped metallicity map of NGC 4710.

\subsection{The origin of the population gradients in NGC 4710}

Simulations show that B/PS bulges can be produced by secular dynamical processes driven by a bar. Bulges formed in this way often show an underlying X-shaped structure in unsharp-mask images. This is the case for NGC 4710, as shown in Fig. 3, where the arms of the X can be clearly identified. The unsharp-masked MUSE image was constructed dividing the original image by a smoothed one that is obtained from convolving the original image with a Gaussian with $\sigma=15$ pixels $(3 \operatorname{arcsec})$. Fig. 3 also shows a more detailed view of the mass-weighted metallicity map which appears more pinched/peanut-shaped than the flux distribution off the mid-plane.

Debattista et al. (2016) predicted that kinematic fractionation produces a B/PS bulge which has a metallicity distribution that is even more peanut-shaped than the density itself. The metallicity map of their simulation is shown in the right-hand panel of Fig. $3 .{ }^{4}$ This shows a remarkable qualitative similarity to the metallicity map of NGC 4710 , both in terms of shape and of vertical metallicity gradient. This metallicity distribution in the bulge is a consequence of the anticorrelation between in-plane velocity dispersion and metallicity at the time of bar formation. The metal-rich populations with low radial velocity dispersion are driven by the bar to a vertically thick peanut-shaped bulge, whereas the hotter, more metal-poor populations become vertically thicker with a more boxy, rather than peanut shape.

There is also a separate inner peanut-shaped, higher metallicity region at $r \lesssim 0.5 \mathrm{kpc}$ in the simulation $([\mathrm{Fe} / \mathrm{H}]>0.1$ dex region in the right-hand panel of Fig. 3 which is not seen in NGC 4710. However, as this is the region dominated by the (inner) disc, this feature does not affect our conclusions.

The detection of the strong peanut-shape in the metallicity distribution of the B/PS bulge of NGC 4710, which is also homogenously old, represents strong evidence in favour of kinematic fractionation having shaped its bulge.

\footnotetext{
${ }^{4}$ For ease of comparison, all metallicities in the simulation are shifted by -0.35 dex. Because here we are interested in the trends, not the absolute values, this shift has no effect on our analysis.
}

\section{CONCLUSIONS}

We have shown for the first time, using VLT-MUSE IFU observations of the edge-on galaxy NGC 4710, that at least some B/PS bulges show a distinct peanut shape in the metallicity distribution, with the peanut shape more pronounced than in the density itself. This behaviour was predicted by the kinematic fractionation model of B/PS bulge growth (Debattista et al. 2016) and is a direct consequence of the redistribution of stellar orbits by the bar based on their in-plane velocities at the time of bar formation.

The peanut-shaped bulge metallicity distribution in NGC 4710 is homologous with the X-shaped structure of the MW's bulge, which is traced by the double-peaked LOS distribution of metal-rich stars, but not by metal-poor ones (Ness et al. 2012; Vásquez et al. 2013). The fact that kinematic fractionation is able to produce vertical metallicity gradients as strong as those observed in NGC 4710, and comparable to those in the MW (Gonzalez et al. 2016), implies that vertical metallicity gradients in B/PS bulges do not require an additional, accreted bulge component (i.e. a classical bulge). The absence of a classical bulge in NGC 4710, as determined from photometric fitting, supports the interpretation that the MW also lacks a classical bulge.

Therefore, the properties of the MW's bulge, once considered puzzling, now appear a rather natural outcome of purely secular evolution. We have shown here that such evolution also occurred in NGC 4710, hinting that many of the MW bulge's properties may be very common amongst B/PS bulges. Our results suggest a number of fruitful next tests of kinematic fractionation. Most obviously, the number of bulges with studied metallicity maps needs to be expanded, including in non-B/PS bulges. Kinematic fractionation also predicts that $\mathrm{B} / \mathrm{PS}$ bulges should exhibit a peanut shape and vertical gradient also in the age (Debattista et al. 2016). The age resolution of our spectra is not sufficient at the old ages we find in NGC 4710 to detect these variations in the age. Searching for these features in a larger sample of B/PS bulges would provide additional support that kinematic fractionation has sculpted the B/PS bulges observed today.

\section{ACKNOWLEDGEMENTS}

This Letter is based on observations collected at the ESO La SillaParanal Observatory within MUSE science verification program 
60.A-9307(A). We are grateful to Adrien Guerou for great advice and comments for the analysis of MUSE cubes and Ruben SanchezJanssen for useful discussions. VPD is supported by STFC Consolidated grant \#ST/M000877/1. MN is funded by the European Research Council under the European Union's Seventh Framework Programme (FP 7) ERC Grant Agreement \#321035. We acknowledge support from the ESF Exchange Grant (number 4650) within the framework of the ESF Activity entitled 'Gaia Research for European Astronomy Training'. The star-forming simulation used in this Letter was run at the High Performance Computing Facility of the University of Central Lancashire. VPD wishes to express his sincere thanks to the Pauli Institute, the University of Zürich, and MPIA Heidelberg for hosting his sabbatical during which important work for this Letter was completed.

\section{REFERENCES}

Athanassoula E., 2016, in Laurikainen E., Peletier R. F., Gadotti D. A., eds, Astrophysics and Space Science Library, Vol. 418, Galactic Bulges. Springer-Verlag, Berlin, p. 391

Bacon R. et al., 2001, MNRAS, 326, 23

Bacon R. et al., 2010, in McLean I. S., Ramsay S. K., Takami H., eds, Proc. SPIE Conf. Ser. Vol. 7735, Ground-based and Airborne Instrumentation for Astronomy III. SPIE, Bellingham, p. 773508

Brooks A., Christensen C., 2016, in Laurikainen E., Peletier R. F., Gadotti D. A., eds, Astrophysics and Space Science Library, Vol. 418, Galactic Bulges. Springer-Verlag, Berlin, p. 317

Buta R. J. et al., 2015, ApJS, 217, 32

Cappellari M., Copin Y., 2003, MNRAS, 342, 345

Cappellari M., Emsellem E., 2004, PASP, 116, 138

Cappellari M. et al., 2011, MNRAS, 413, 813

Cappellari M. et al., 2013, MNRAS, 432, 1862

Cenarro A. J., Cardiel N., Gorgas J., Peletier R. F., Vazdekis A., Prada F., 2001, MNRAS, 326, 959

Cole D. R., Debattista V. P., Erwin P., Earp S. W. F., Roškar R., 2014, MNRAS, 445, 3352

Debattista V., Ness M., Gonzalez O. A., Freeman K., Zoccali M., Minniti D., 2016, MNRAS, preprint (arXiv:1611.09023)
Dékány I., Minniti D., Catelan M., Zoccali M., Saito R. K., Hempel M., Gonzalez O. A., 2013, ApJ, 776, L19

Gadotti D. A., 2012, Astron. Astrophys. Trans., 27, 221

Girardi L., Bressan A., Bertelli G., Chiosi C., 2000, A\&AS, 141, 371

Gonzalez O. A., Rejkuba M., Zoccali M., Valent E., Minniti D., Tobar R. 2013, A\&A, 552, A110

Gonzalez O. A. et al., 2016, A\&A, 591, A7

Guérou A., Emsellem E., Krajnović D., McDermid R. M., Contini T., Weilbacher P. M., 2016, A\&A, 591, A143

Hopkins P. F. et al., 2010, ApJ, 715, 202

Johnson C. I., Rich R. M., Kobayashi C., Kunder A., Pilachowski C. A., Koch A., de Propris R., 2013, ApJ, 765, 157

Kormendy J., Kennicutt R. C., Jr, 2004, ARA\&A, 42, 603

Laurikainen E., Salo H., 2016, in Laurikainen E., Peletier R. F., Gadotti D. A., eds, Astrophysics and Space Science Library, Vol. 418, Galactic Bulges. Springer-Verlag, Berlin, p. 77

Lütticke R., Dettmar R.-J., Pohlen M., 2000, A\&A, 362, 435

Ness M. et al., 2012, ApJ, 756, 22

Ness M. et al., 2013, MNRAS, 430, 836

Ness M., Debattista V. P., Bensby T., Feltzing S., Roškar R., Cole D. R., Johnson J. A., Freeman K., 2014, ApJ, 787, L19

Quillen A. C., Minchev I., Sharma S., Qin Y.-J., Di Matteo P., 2014, MNRAS, 437,1284

Raha N., Sellwood J. A., James R. A., Kahn F. D., 1991, Nat, 352, 411

Salpeter E. E., 1955, ApJ, 121, 161

Shen J., Rich R. M., Kormendy J., Howard C. D., De Propris R., Kunder A., 2010, ApJ, 720, L72

Vásquez S. et al., 2013, A\&A, 555, A91

Vazdekis A., Sánchez-Blázquez P., Falcón-Barroso J., Cenarro A. J., Beasley M. A., Cardiel N., Gorgas J., Peletier R. F., 2010, MNRAS, 404, 1639

Vazdekis A., Ricciardelli E., Cenarro A. J., Rivero-González J. G., DíazGarcía L. A., Falcón-Barroso J., 2012, MNRAS, 424, 157

Wegg C., Gerhard O., 2013, MNRAS, 435, 1874

Zoccali M., Valenti E., 2016, Publ. Astron. Soc. Astron., 33, e025

Zoccali M., Hill V., Lecureur A., Barbuy B., Renzini A., Minniti D., Gómez A., Ortolani S., 2008, A\&A, 486, 177

This paper has been typeset from a $\mathrm{T}_{\mathrm{E}} \mathrm{X} / \mathrm{L} \mathrm{T}_{\mathrm{E}} \mathrm{X}$ file prepared by the author. 